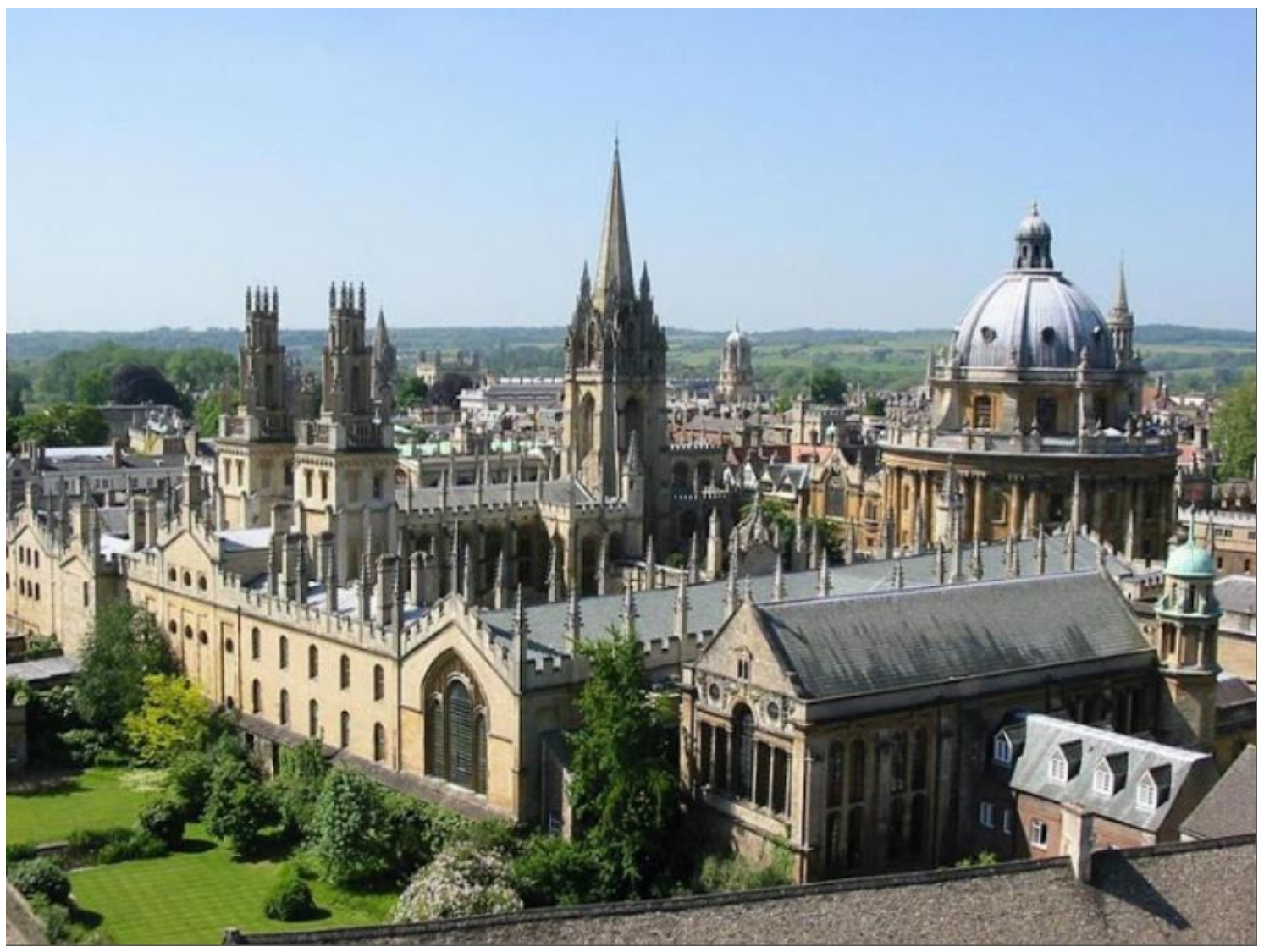

Flow Injection Analysis of Hydrogen Peroxide Using a Horseradish Peroxidase Modified Electrode Detection System.

P. Dominguez; J.M. Fernandez Alvarez; P. Tunon Blanco; R. O'Rennedy and M.R. Smyth

Dublin City University, Glasnevin, Dublin 9, Ireland.

A Flow Injection Analysis (FIA) system is described for the determination of levels of hydrogen peroxide down to $10 \times 10^{-8} \mathrm{M}$ using a horseradish peroxidase modified electrode detection system. The response is based on the enzymatic reduction of hydrogen peroxide in the presence of hydroquinone acting as chemical mediator. 


\title{
Flow-Injection Analysis of Hydrogen Peroxide Using a Horseradish Peroxidase-Modified Electrode Detection System
}

\author{
P. Domínguez, J. M. Fernández Álvarez, P. Tuñón Blanco, R. O’Kennedy \\ and M. R. Smyth \\ Dublin City University, Glasnevin, Dublin 9, Ireland
}

\begin{abstract}
A flow-injection analysis (FIA) system utilizing a horseradish peroxidise-modified amperometric electrode is described. The enzyme was immobilized through adsorption onto a glassy carbon electrode and the system is used to determine hydrogen peroxide at submicromolar levels. The response is based on the enzymatic reduction of hydrogen peroxide in the presence of hydroquinone acting as an electron mediator. The oxidized quinone produced in the enzymatic reaction is reduced at the electrode, and the reduction current was found to be proportional to the concentration of hydrogen peroxide. Calibration curves were found to be linear between $2.5 \cdot 10^{-8} \mathrm{M}$ and $1.0 \cdot 10^{-6}$ $\mathrm{M}$, and the method has a detection limit of $1.0 \cdot 10^{-8} \mathrm{M}$.
\end{abstract}

